to a precise and tedious inspection of its edges and to a careful tactile examination of its base and to check what is discovered by results laboriously gained from like inquiries? Is it not simpler to take a scraping of the affected surface and to submit it to a microscopist, and thus to be spared a method of examination which, although it may not give inal results, yet represents an opportunity of furthering a priceless accomplishment?

Finally, there are cases which present symptoms hard to interpret at any superficial inquiry. Is it worth while in such to undertake an exhaustive critical research and to submit the whole to a trained judgment? The quest would no doubt develop habits of observation and powers of reighing evidence; but the process is slow, and an inquiry carried out in a bacteriological laboratory will clear up all doubts and at the same time dispense with the efforts of a cultured sense. Those, therefore, who are concerned with the education of the surgeon of the future would do well to cherish still this ancient power and to foster a memory of the fact that surgery is, in its very essence, a handicraft, and that in all that he does the surgeon's great endeavour should be to make his own hands self-sufficing.

It is sad to think that this hardly-acquired faculty dies with the possessor of it, and never was this more vividly presented than it has been by the loss which surgery has sustained in the death of Sir James Paget. One can picture the great surgeon composed in his last sleep, and can see the once busy fingers lying lifeless on the white sheet, and then comes the wonder of the wealth of learning, at the exquisite cunning, at the refined sense which lay dead in the dead hand. No written book can hold a tithe of the dainty knowledge which had been mastered by those subtle fingers, and no resord, however laboured or however loving, can tell of the power which once rested in that magic touch. An individual loss does not, however, hinder the general tide of progress. Advance in such a work as ours depends upon the uneventful work of the whole body and is only accentuated by the achievements of the prominent few. The movement is the movement of a multitude in which individuality is, at a distance of time, little to be distinguished and in which personal eminence contributes a smaller factor than the present is ready to acknowledge. Those who stand forth as the leaders of the advance are marely the elect of the common body and the representatives of a wide intellectual franchise. Even he who startles the world as a discoverer has often done little more than give expression to what was already nascent in the multitude. So as one great surgeon after another drops out of the ranks his place is rapidly and imperceptibly filled, and the advancing line moves on with still the same solid and unbroken front. The continuity of progress is undisturbed by the uncertainties of human life, and, as one writer has well expressed it-

"No work begun shall ever pause for death."

\section{A CASE OF MENINGO-MYELITIS WITH} BACTERIOLOGICAL EXAMINATION OF THE SPINAL CORD.

\section{BY GEORGE R. MURRAY, M.A., M.D. CAMB.,} F.R.C.P. LOND.,

HEITE PROFESSOR OF COUPARATIVE PATHOLOGX IN THE UNIVERSTTY F DURHAM; PHYSICIAN TO THE ROYAL INFIRMARY, NEWCASILE;

$$
\text { AN D }
$$

W. HARDCASTLE, M.B. LoND., B.HY. DURH.,

IFMTSSTRATOR OF BACTERIOLOGY IN THE UNIVERSITY OF DURHAM; RESDENT VEDTCAL OFFICER AT THE DISPENSARY, NEWCASTLE,

THE acute onset and rapid course of many cases of meningo-myelitis are very strong indications of the infective nature of the disease. Several cases have been recorded in Which a bacteriological examination of the spinal cord and veninges has revealed the presence of a micro-organism endowed with pathogenic properties. The total number of such cases is small and so we consider that the following case is worthy of being published in spite of the incompleteness of both the clinical record and the bacteriological examination.

A single man, aged 29 years, was admitted into the Newcastle Royal Infirmary on Jan. 3rd, 1899, on account of complete loss of power and sensation in both lower limbs. A month previously when in his ordinary good health he was wakened in the middle of the night and called out to help at a fire in the village in which be lived. He quickly dressed, went out, and took his place in a line of men which was formed to pass buckets of water up to the scene of the fire. He worked hard at this for several hours during which he got hot and exhausted in his arms and the upper part of his body, while his feet and legs were wet and chilled by the water which was accidentally spilled on them in rapidly passing on the buckets filled with water. After this he was seized with a severe pain in the lumbar region and the legs felt cold and numb. The pain was relieved by the application of blisters, but the sensation of cold and numbness persisted, being accompanied by a steadily increasing loss of muscular power, so that by the end of a week both legs were completely paralysed. This was shortly followed by some difficulty in micturition with constipation. At the end of a fortnight from the commencement of the illness there was retention of urine with loss of control over the rectum. Since then the regular use of a catheter had been necessary and the fæces had been passed unconsciously. The family history was unimportant and the only previous illnesses from which the patient had suffered were pleurisy and influenza.

On admission into the Infirmary a month after the beginning of his illness the patient lay on his back in bed complaining of a sensation as if a girdle were tied round him midway between the ensiform cartilage and the unbilicus and of pain in the lower part of the abdomen which was increased when the bladder became, full. There was complete paralysis of both lower limbs and of the abdominal muscles as high as the umbilicus, combined with loss of all forms of sensation up to the same level. There was no control of the bladder, which if not emptied by means of a catheter filled and then overflowed. The control of the rectum was also lost, the freces being passed unconsciously into the bed. The motor power of the muscles of the arms and neck and of the trunk above the level of the umbilicus was retained and the sensation of the skin covering these parts was not affected. The plantar and superficial abdominal reflexes up to the level of the umbilicus were absent, but the cremasteric reflex was still present. There was no ankle clonus; the knee-jerks were still present but the response was very slight and feeble. There was a large bed-sore over the sacrum. The urine was acid in reaction, but had a very foul smell and contained both blood and pus. The temperature on admission was $103 \cdot 4^{\circ} \mathrm{F}$.

During the first few days that the patient was in the Infirmary the condition remained much as above and the temperature fell to normal (vide Fig. 1). On the evening of the 11th there was a severe rigor, the temperature rapidly rising to $105^{\circ}$ at 8.30 P.M. On the following morning the temperature was normal again, the pulse was 150 , and the respirations were 28 . The patient complained of difficulty in breathing and of pain in the lower part of the left side of the chest and at the epigastrium. The upper part of the chest expanded freely, but the expansion of the lower part was deficient. There was general tenderness on pressure all over the abdomen, which was most marked over the kidneys. The knee-jerks were still present and a slight plantar reflex could be obtained on the left side but not on the right. On Jan. 15th the patient began to hiccongh and this continued for a week. On some days the hiccough only ceased for one hour out of every three or four and the hypodermic injection of the fifth of a grain of morphia only controlled it for a couple of hours at a time. On the 17th the upper limit of anæsthesia was found to run midway between the umbilicus and the centre of Poupart's ligament, at which level it subsequently remained. The pulse was 82 . The abdomen was distended but resonant all over. The left plantar, the cremasteric, and the patellar reflexes could still be obtained, but the response was very feeble. The patient gradually got worse and on Jan. 24 th the muscles of both legs were found to be much wasted, soft, and toneless. The plantar reflexes had disappeared and no knee-jerks could be obtained. After this the patient gradually sank and died at 11.30 A.M. on Jan. 27th, seven and a half weeks after the commencement of the illness.

Necropsy.-Unfortunately, no full post-mortem examination was allowed and leave could only be obtained to open the spinal canal and remove the spinal cord. This was done on Jan. 27th at 5.30 P.M., six hours after death. As soon as the spinal canal had been opened some of the lymph which was 
FIG. 1.

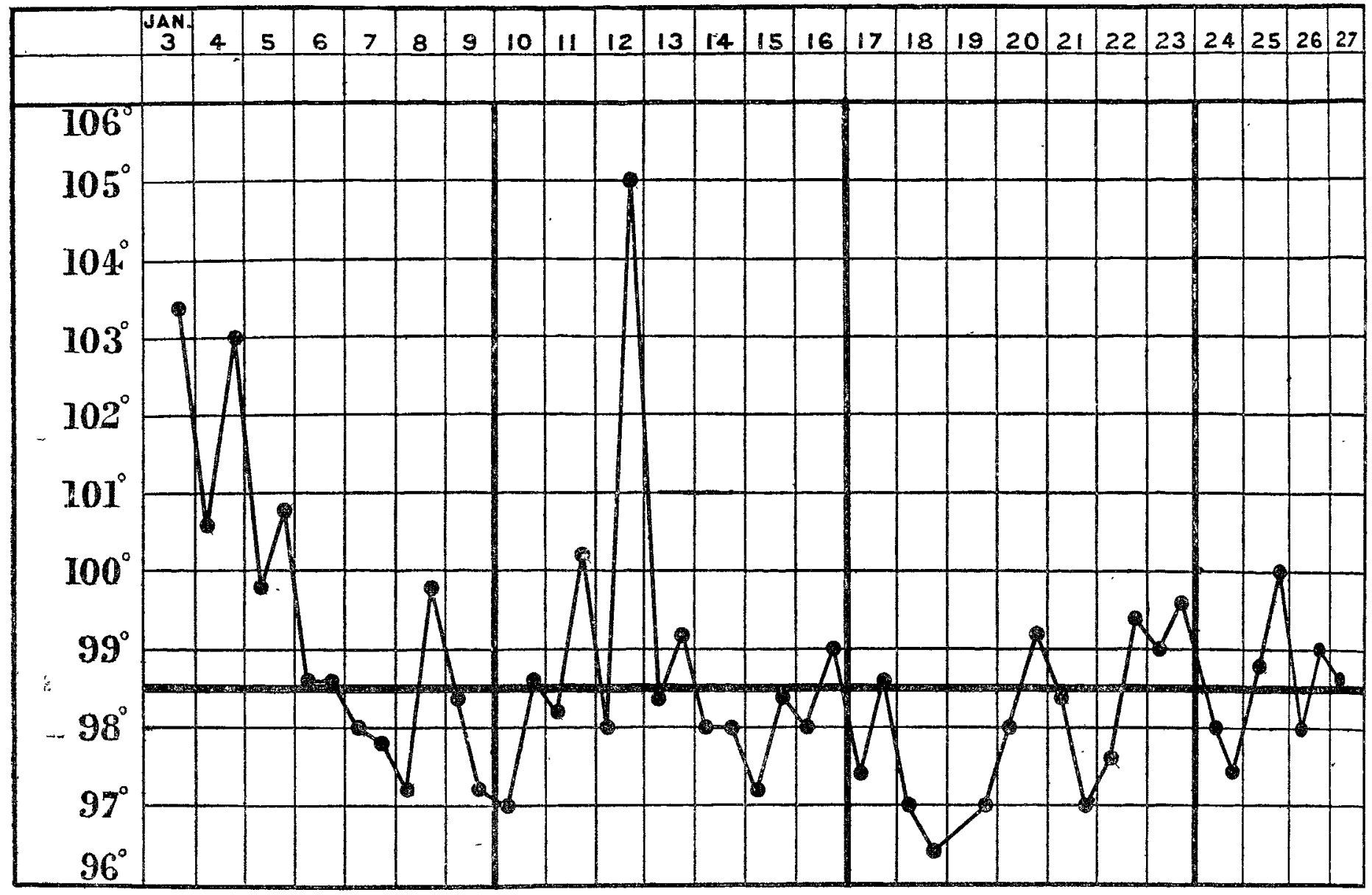

Temperature chart of a case of meningo-myelitis.

lying on the surface of the dura mater in the upper dorsal region was raised from the dura mater by means of a pair of sterilised forceps, a platinum wire was pushed up between the two, and stroke cultures made on agar-agar and on serum-agar tubes. In this manner inoculations were made direct from the under surface of the layer of lymph, where it lay in contact with the dura mater. The dura mater was then opened with sterilised instruments and stroke cultures on agar were made in the same way from the surface of the pia mater in the mid-dorsal regions. Transverse cuts were then made across the cord with a sterilised knife in the dorsal region on a level with the roots of the fourth and fifth dorsal nerves, and also in the lumbar enlargement. A sterilised platinum loop was then pushed into the substance of the cord itself from the freshly.cut surface in each of these situations, and stroke cultures were made on agar-agar and on serum-agar tubes. After the spinal cord had been removed it was found that there was a communication between the spinal canal and the large bed-sore over the sacrum. This communication may, however, have been accidentally made at the time of the necropsy.

All the tubes were placed in the warm incubator at $37^{\circ} \mathrm{C}$. on the same evening. After hardening, sections of the spinal cord from the dorsal region were cut and stained with picrocarmine and then by Weigert's modification of Gram's method. It was found that if the washing with aniline oil was carried on until all the violet colour had disappeared from the substance of the cord and pia mater the microorganisms were also decolourised, but when the aniline oil was removed while the violet colour was still distinguishable to the naked eye in the pia mater the micro-organisms were well stained. In sections thus prepared large numbers of a small oval bacillus were seen in the pia mater. In some places the bacilli were stained at the extremities and clear in the centre, closely resembling diplococci. The bacilli were lying singly or in irregular chains. One or two longer rod-shaped forms were seen, but with this exception no other forms were found. The oval bacilli were also seen in small groups here and there in the white substance of the cord itself and separated from the pia mater, but the further from the surface of the cord the fewer the groups became.

Cultures. - The tubes were inoculated and placed in the incubator at $37^{\circ} \mathrm{C}$., as already mentioned, on the evening of
Jan. 27th. No visible growth had appeared on the 28th, bot on the morning of the $29 \mathrm{th}$, after the tubes had been about 40 hours in the incubator, colonies had appeared in all the tubes except in one tube of serum-agar inoculated from the substance of the lumbar enlargement. In this tube, how ever, a growth had also developed by Feb. 1st. Ten tubes in all had been inoculated from the meninges and from the cord itself. In eight of these the growth proved on microscopical examination to be a pure culture of a short oval bacillus. The other two showed similar growths, but in one of them it was mixed with a few larger and longer bacilli, and in the other with some chains of streptococci. As far as naked-ey appearances went the growth in each of the pure cultures had the same appearance. On microscopical examination slight differences in appearance were found in the different cultures. In all the bacillus was short, the length not being more than double the breadth, though there was some difference in the actual size in different specimens. Slight differences were also due to different degrees of staining; in some cases the oval bacilli were stained all through, in others the ends only were stained and the centre clear, so that they resembled diplo. cocci. We consider, however, that they are rightly described as short oval bacilli with polar staining. The appearance is well shown in Figs. 2 and 3 , micro-photographs which were kindly taken for us by Mr. H. R. Spitta. Fig. 2 was taken from a film prepared from a pure culture obtained directly from the substance of the spinal cord on a level with the roots of the fourth dorsal nerve. Fig. 3 was taken from a film preparation from a pure culture obtained from a guinea-pig' blood after subcutaneous inoculation with a broth sub-culture obtained from the growth shown in Fig. 2. In the banging drop the bacillus was seen to be motile. It grew readily a $37^{\circ} \mathrm{C}$. on nutrient agar. Isolated colonies on agar at the end of 40 hours in the incubator were small and translucent and resembled minute droplets of condensed moisture. They became rather milky in appearance when older. In a uniform stroke culture a translucent slightly opalescent laser ras found at the end of 48 hours. At ordinary temperatures it grew in gelatin which it liquefied.

Inoculations. - The pathogenic properties of the bacillus were tested in a guinea-pig and in a rabbit. For this purpose a tube of nutrient broth was inoculated from the agar 
culture obtained from the substance of the spinal cord in the upper dorsal region and incubated at $37^{\circ} \mathrm{C}$. for 24 hours. At the end of this time an abundant growth of bacilli had taken place in the broth. One cubic centimetre of this broth culture was injected with the usual antiseptic precautions beneath the skin of the inner side of the thigh of a brown male rabbit, and a similar quantity of the same culture was also injected into a red-and-white male guinea-pig in the same situation. On the following day the guinea-pig looked ill, the temperature in the morning being $100.6^{\circ} \mathrm{F}$, and in the evening $99 \cdot 4^{\circ}$. On the next morning it was found dead in

FIG 2

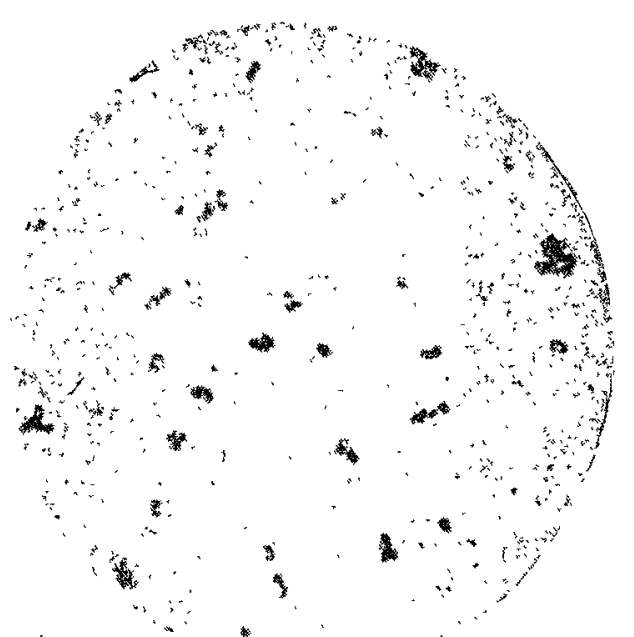

Film preparation of culture obtained from substance of spinal cord.

the cage. No naked-eye changes were found at the post mortem examination. Films were prepared from the blood from the heart and stained. Agar tubes were inoculated from the blood in the heart, and films were prepared from the cultures which developed. On microscopical examination of the blood a few widely-scattered micro-organisms which resembled diplococci without any capsule were found. In the hot incubator a pure culture of the original bacillus developed on agar, so that the micro-organisms in the blood were really bacilli with polar staining and not diplococci, the actual form being more easily seen in pure cultures than in the blood itself.

FIG. 3.

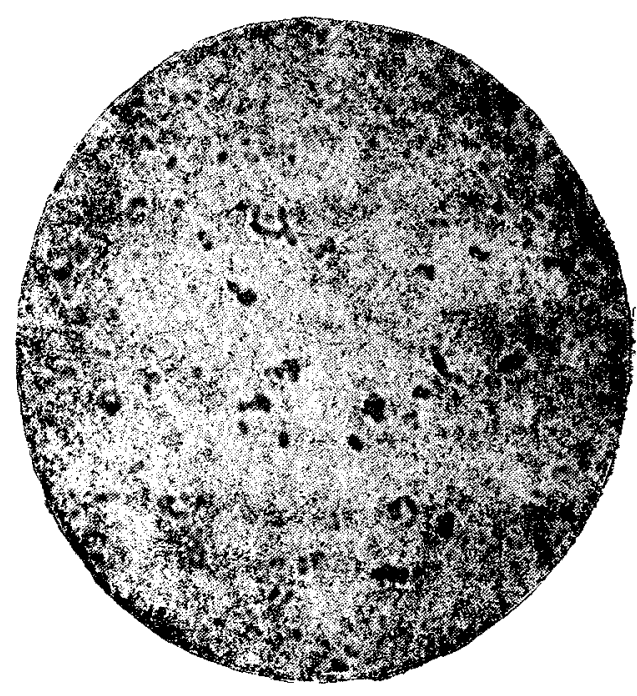

Film preparation of culture obtained from blood of guinea-pig after inoculation.

Fig. 3 shows the specimen prepared from the agar culture obtained from the blood of the guinea-pig. In the case of the rabbit, on the day after the inoculation the temperature in the morning was $102^{\circ}$ and in the evening $104^{\circ}$. The animal was very quiet and the respiration was rapid and laboured. The following morning the temperature had fallen to $100.2^{\circ}$ and a swelling had formed at the seat of the inoculation. After this the temperature only varied between $100^{\circ}$ and $101^{\circ}$. On the fourth day after the inoculation the right side of the scrotum was swollen and the rabbit was killed. The right tunica vaginalis was distended with thin serous fluid and the right testicle was swollen. A slough had formed at the seat of inoculation. Tubes were inoculated from the heart blood and from the tunica vaginalis. No colonies developed from the blood but an abundant pure growth of the original bacillus developed in the tube inoculated from the tunica vaginalis. In the guinea-pig, therefore, the subcutaneous inoculation of a pure culture of the bacilli produced a rapidly fatal general infection without local reaction, whereas in the rabbit there was no general infection, but a local reaction, with sharp rise of temperature, limited to the neighbourhood of the inoculation. No nervous symptoms were observed in either of the animals.

The characters of the micro-organisms may be shortly summarised as follows. A short oval motile bacillus which stains readily by aniline dyes, the stain in many cases showing a much stronger affinity for the ends than for the centre of the bacillus. It can with care be stained by Gram's method and was found in large numbers in the pia mater and also in lesser numbers in the substance of the cord. Pure coltures of the bacillus were obtained both from the meninges and also from the substance of the cord itself. Subcutaneous inoculation caused rapid general infection in the guinea-pig and a local infection in the rabbit. The bacillus grows readily on the ordinary nutrient media in both the hot and cool incubators. It liquefies gelatin.

In this case we have a clear history of an acute onset of the meningo-myelitis following an exposure to cold and wet combined with fatigue. The similarity to the sequence of events often observed in the development of an attack of acute lobar pneumonia is striking, so that it seems very probable that the myelitis, like a pneumonia, was the result of an infection occurring as a result of a temporary lowering of resistance. This view is strongly supported by the presence of a micro-organism in the meninges and spinal cord, which we have shown was undoubtedly possessed of pathogenic properties.

Micro-organisms have been found and cultivated in several cases of acute meningo-myelitis, notably in the case so well recorded by Dr. T. Buzzard and Dr. Risien Russell. ${ }^{1}$ They obtained a culture of a diplococcus in one tube which was inoculated from the meninges in the lumbar region, the other tubes showing no growth. The organisms seem to have been less numerous than in our case, as growth developed in all the tubes we inoculated. Similar diplococci were seen in stained sections in the exudation, in the meninges, and in the substance of the cord, just as in our case. The organism in our case differs from that found by Buzzard and Russell in that it more nearly resembled bacillus with polar staining than a diplococcus-a difference that is perhaps more apparent than real-it liquefies gelatin, and can be stained in sections by Gram's method. It differs from Weichselbaum's diplococcus intracellularis in appearance and, unlike it, grows in the cool incubator and does not appear within the cells of the exudate.

In Dr. E. F. Trevelyan's case ${ }^{2}$ a diplococcus was obtained from the pus outside the dura mater and also from a softened patch in the cord; the bacteriological examination was, however, not carried further as the micro-organisms died in a few days.

The value of the bacteriological examination in our case is, of course, much diminished by the fact that the bed-sore was in such close proximity to, if not in actual communication with, the spinal canal, but we consider that if the infection of the cord had been secondary to the foul bed-sore the cultures would have been mixed and not pure in all except two tubes, as was the case. The evidence we at present possess goes a long way to show that acute meningomyelitis is probably an infective disease, and although it cannot as yet be attributed to any single organism the bacteria which have been found in the recorded cases appear to have certain characters in common.

Newcastle.

1 Transactions of the Clinical Society of London, vol. xxxi., p. 185. 2 Brain, Part lxxxvili., 1899, p. 607.

Local Sanitary Authorities and the MilkSUPPLY.-The bacteriological examinations conducted by the St. Pancras Vestry have proved that out of 50 samples only 16. were normal, five contained tubercle bacilli, and the remainder from various causes were not fit for human consumption. 


\section{A BLOOD REACTION IN DIABETES MELLITUS; ITS CAUSE AND DIAGNOSTIC VALUE.}

By R. T. WILLIAMSON, M.D., F.R.C.P. LOND., PHYsiclai to The axcoats Hospital, MaNChRSTER, aNd assistay LRCTURER OX MEDICINE AT OWENS COLLEGE.

IN 1896 I recorded ${ }^{1}$ a reaction which $I$ believe to be peculiar to the blood of patients suffering from diabetes mellitus. A drop of blood taken from the finger of a diabetic patient decolourises a warm alkaline solution of methylene blue, when mixed in certain proportions, whilst blood from healthy persons or from individuals suffering from other diseases has no such action when mixed in the same proportion. The reaction is due to the greater reducing power of diabetic blood and is obtained as follows. At the bsttom of a small, narrow test-tube is placed a drop of water -40 cubic millimetres. To this are added 20 cubio millimetres of blood taken from the finger of the diabetic patient. Then 1000 cubic millimetres (one cubic centimetre) of a watery solution of methylene blue ( 1 in 6000) and finally 40 cubic millimetres of liquor potassæ (B. P.) are added. Into a similar tube are placed 20 cubic millimetres of normal blood with the same proportions of methylene blue and liquor potassæ. The mixed fluids in each tube will have a fairly deep blue or bluish-green colour. Both small test-tubes are placed in a beaker or very large wide test-tube containing water and the water is heated and kept boiling for four minutes. At the end of this time the fluid in the small testtube containing the diabetic blood will have lost its blue colour and become dirty yellow, whilst that in the tube containing non-diabetic blood will remain blue or bluish-green. (The exact quantities of the fluid used may be measured by the small tubes supplied with the hæmocytometer and hæmoglobinometer of Gowers.)

I have examined the blood in 43 cas as of diabetes mellitus and have always obtained the reaction mentioned. I have examined the blood in 100 cases of othor diseases of the most varied forms and also the blood of healthy persons on innumerable occasions, but, when the fluids have been mixed in the proportion above stated, have never obtained the reaction. My results have been confirmed in Germany by Loe wy, ${ }^{2}$ Goldscheider, ${ }^{3}$ and R. Müller ${ }^{4}$; in France by Le Goff, ${ }^{5}$ P. Marie, Lyonnet, ${ }^{6}$ and Lépine : in Italy by Baduel and Castellani, ${ }^{7}$ Ferrannini, ${ }^{8}$ and Lucibelli ${ }^{9}$; and in America by Futscher ${ }^{10}$ (in Professor Osler's clinic). Further observations which $I$ have made indicate the cause and diagnostic value of the reaction.

CAUSE OF THE ReACTION.

1. This reaction, peculiar to diabetic blood, is evidently due to the presence of some substance in solution in the blood serum. Large quantities of blood obtained post mortem from the internal jugular vein of three cases of diabetes mellitus were allowed to stand until the serum separated on the surface and the red corpuscles sank to the bottom of the vessel. In each case the blood serum, free from red corpuscles, gave the reaction-i.e., decolourised methylene blue in the proportion given above-whilst the serum obtained in a similar manner from non-diabetic blood gave no reaction (in the proportions mentioned).

2. Blood serum from a diabetic subject loses the power of giving the reaction after it is submitted to the action of yeast. In three cases of diabetes mellitus large quantities of blood were taken post mortem from the internal jugular vein and allowed to stand for 24 hours until the serum had separated.

1 Brit. Mer. Jour., Sept. 19th, 1896.

2 Berliner Klinische Wochenschrift, 1897, No. 46, and Fortschritte der Medicin, 189

3 Deutsche Medicinische Zeitung. 1898.

4 Münchener Medicinische Wochenschrift, 1899, No. 25

Bulletin et Mémoire de la Société Médicale des Hôpitaux de Paris, 1897, No. 16 and No. 17; Sur certaines Réactions Chromatiques du Sang dans le Diabète Sucré, Paris, 1897, pp. 44 to 81.

6 Lyon Médical, 1896, tome lxxxiii., p. 187.

Medicz, 1898 No. 10. Medicin, 1898, No. 28 .

Iedicin, 1898, No. 28. $27 \mathrm{th}, 1902$.

10 Philadelphia Medical Journal, 1998, No. 7
In each case two test-tubes of the usual size were half filled with the diabetic blood serum. To one test-tube ordinary German yeast was added, to the other no yeast was added. Both were kept in a warm place, side by side, for 48 hours, the mouth of each tube being plugged by cotton-wool. A the end of 48 hours the serum in the test-tube containing the yeast gave no reaction with methylene blue (according to the method above described), whilst 20 cubic millimetres of the serum in the tube which contained no yeast gave the reaction readily. The same results were obtained by the blood serum in all of the three cases of diabetes. The diabetic serum which had been submitted to the action of yeast and which did not decolourise methylene blue gave no reaction for sugar with Fehling's solution; whilst the diabetic serum to which no yeast had been added and which continued to give the reaction with methylen blue above described, gave a slight but distinct reaction for sugar with Febling's solution. The failure of the diabetic serum, after the action of yeast, to give the methylene-blue reaction was not due simply to the presence of the yeast. cells in the fluid, since diabetic serum when mixed with yeast and tested immediately gave the metbylene-blue reaction distinctly. Hence we may conclude that the diabetic serum loses its power of giving the methylene-blue reaction, owing to the fermenting action of the yeast fungus, which in the course of 27 or 48 hours destroys some substance in the serum.

3. After the proteids of diabatic blood or serum have been separated, by boiling with crystalline sulphate of soda and filtering, the filtrate-gives the methylene-blue reaction. A number of observations were made on the blood obtained post mortem from the internal jugular vein in a case of diabetes. In each examination an ounce or more of the blood or serum was mixed with an equal quantity of crystalline sulphate of soda and the mixture was boiled until the froth above the clot became white and all red specks disappeared. In every examination 20 cubic millimetres the clear filtrate gave the methylene-blue reaction abore described. When non-diabetic blood was treated with sodium sulphate in the same way the filtrate failed to give the methylene-blue reaction.

4. After the separation of the proteids of diabetic blood the power of giving the methylene-blue reaction is destroyed by the action of yeast. In another experiment, two testtubes were half filled with the clear filtrate, obtained after separating the proteids of diabetic blood by boiling with sodium sulphate, as just described. To the fluid in one testtube a little German yeast was added, while to that in the other tube no yeast was added. The mouth of each tube was plugged with cotton-wool and the tubes were kept in a warm place for 48 hours: Then the fluid in each tube was examined for the methylene-blue reaction abore described. 20 cubic millimetres of the fluid from the testtube of filtrate to which yeast had been added gave no reaction ; 20 cubic millimetres of the fluid from the test-tube to which no yeast had been added gave the reaction mark edly. The fluid in the test-tube to which yeast had been added gave no reaction for sugar either with Fehling's solu tion or phenyl-hydrazin, whilst the fluid in the other test tube, to which no yeast had been added, gave a reaction for sugar both with Fehling's solution and phenyl-hydrazin

5. It is well known that glucose bas the power of reducing a warm alkaline solution of methylene blue. 20 cubic milli metres of a watery solution of glucose (5 in 1000) gave distinct reaction with the methylene-blue test; but 20 cubic millimetres of a 2.5 in 1000 solution did not. When 20 cubic millimetres of the 2.5 in 1000 solution of glucose were added to 20 cubic millimetres of normal blood, the mixture decolourised the alkaline methylene blue like diabetic blood but 20 cubic millimetres of the blood alone, or of the 2.5 in 1000 glucose solution alone, failed to give the metbylene-blue reaction.

6. Though non-diabetic blood does not give the methyleneblue reaction in the proportions stated at the commencement of this article, it has nevertheless some reducing action in larger quantities. In performing the metbylene-blue blood test it is always necessary to measure accurately the quantity of blood employed ( 20 cubic millimetres), since the diabetic reaction depends on the greater reducing power of the diabetic blood. As already stated, I have never obtained the reaction when 20 cubic millimetres of blood were employed. in any condition except diabetes mellitus. But with norms blood a reaction may be obtained when 60 cubic milli metres of blood are employed in performing the test; also 